K. Miyake and H. Opolka

Nagoya Math. J.

Vol. 125 (1991), 1-14

\title{
ON NILPOTENT EXTENSIONS OF ALGEBRAIC NUMBER FIELDS I
}

\author{
KATSUYA MIYAKE AND HANS OPOLKA
}

\section{Introduction}

The lower central series of the absolute Galois group of a field is obtained by iterating the process of forming the maximal central extension of the maximal nilpotent extension of a given class, starting with the maximal abelian extension. The purpose of this paper is to give a cohomological description of this central series in case of an algebraic number field. This description is based on a result of Tate which states that the Schur multiplier of the absolute Galois group of a number field is trivial. We are in a profinite situation throughout which requires some functorial background especially for treating the dual of the Schur multiplier of a profinite group. In a future paper we plan to apply our results to construct a nilpotent reciprocity map.

\section{§1. Central extensions and Schur multipliers}

Let $k$ be an algebraic number field of finite degree over the rationals $\mathbf{Q}$, and let $k^{\mathrm{ab}}$ (resp. $k^{\mathrm{nil}}$ ) be the maximal abelian (resp. nilpotent) extension of $k$ in the algebraic closure $\overline{\mathbf{Q}}$ of $\mathbf{Q}$. For each positive integer $c$ denote by $k^{(c)} / k$ the maximal nilpotent extension of class (at most) $c$. Hence $k^{(1)}=k^{\mathrm{ab}}$ and $k^{\mathrm{nil}}=\bigcup_{c=1}^{\infty} k^{(c)}$. For convenience we set $k^{(0)}=k$. Put $G^{c}=$ $\operatorname{Gal}\left(k^{(c)} / k\right)$ and $N^{c}=\mathrm{Gal}\left(k^{(c)} / k^{(c-1)}\right) ; N^{c}$ is a closed normal subgroup of $G^{c}$ which is contained in the center $Z\left(G^{c}\right)$. Therefore we have a central extension of Galois groups

$$
1 \longrightarrow N^{c+1} \longrightarrow G^{c+1} \longrightarrow G^{c} \longrightarrow 1 \text {. }
$$

We furnish the rational torus group $T=\mathbf{Q} / \mathbf{Z}$ with the discrete topology and consider it as a Galois module with trivial action.

Proposition 1. For each $c \geqq 1$, the compact group $N^{c+1}$ is canonically

Received July 6, 1988. 
isomorphic to the Pontrjagin dual of the Schur multiplier $H^{2}\left(G^{c}, T\right)$ of $G^{c}$.

Proof. Put $\mathscr{S}=\operatorname{Gal}(\overline{\mathbf{Q}} / k)$ and $\mathfrak{N}^{c}=\operatorname{Gal}\left(\overline{\mathbf{Q}} / k^{(c)}\right)$. Then we have $G^{c}=$ $\left(s / \Re^{c}\right.$, and the exact Hochschild-Serre sequence

$$
\left.\operatorname{Hom}(\circlearrowleft), T) \stackrel{\text { res }}{\longrightarrow} \operatorname{Hom}\left(\mathfrak{N}^{c}, T\right)^{\leftrightarrow} \stackrel{\tau^{c}}{\longrightarrow} H^{2}\left(G^{c}, T\right) \longrightarrow H^{2}(\&), T\right),
$$

where $\tau^{c}$ is the transgression. The last term $H^{2}(\mathscr{S}, T)$ vanishes by a well known result of Tate; see e.g. [Se], $\S 6$. Therefore $H^{2}\left(G^{c}, T\right)$ is isomorphic to the cokernel of the restriction map which is naturally identified with $\operatorname{Hom}\left(\mathfrak{N}^{c} /\left[\mathfrak{N}^{c}, \mathbb{S}\right], T\right)$. By definition we have $\left[\mathfrak{N}^{c}, \mathbb{S}\right]=\mathfrak{N}^{c+1}$ and $\mathfrak{N}^{c} / \mathfrak{N}^{c+1}=$ $N^{c+1}$. This shows that $H^{2}\left(G^{c}, T\right)$ is isomorphic to $\operatorname{Hom}\left(N^{c+1}, T\right)$. Taking the dual groups we immediately obtain the proposition.

\section{§ 2. The dual of the Schur multiplier}

In this paper, $H^{2}(G, T)$ and its dual for a Galois group $G$ of an infinite algebraic extension plays an important role. When $G$ is finite, $H^{2}(G, T)$ is a finite abelian group and isomorphic to its dual although not canonically. When $G$ is an infinite profinite group, however, $H^{2}(G, T)$ is different from its dual. So it seems worthwhile to give a brief survey on the dual of $H^{2}(G, T)$ for a profinite group $G$.

Let $G$ be a profinite group and suppose that we have a presentation $G=F / R$ with a free profinite group $F$ and its closed normal subgroup $R$ which is generated by the relations in $G$. Associated to the exact sequence

$$
1 \longrightarrow R \longrightarrow F \stackrel{\rho}{\longrightarrow} G \longrightarrow 1
$$

the transgression gives an exact sequence

$$
\operatorname{Hom}(F, T) \stackrel{\text { res }}{\longrightarrow} \operatorname{Hom}(R, T)^{F} \stackrel{\tau}{\longrightarrow} H^{2}(G, T) .
$$

THEOREM 1. The transgression induces an isomorphism

$$
\tau: \operatorname{Hom}(R \cap[F, F] /[R, F], T) \stackrel{\sim}{\longrightarrow} H^{2}(G, T) .
$$

Proof. It is easily seen that the cokernel of the restriction map can be identified with

$$
\operatorname{Hom}(R \cap[F, F] /[R, F], T) .
$$

To show that $\tau$ is surjective, we can slightly modify the method of 
[Ka], pp. 47-48. Let $(\varphi)$ be an element in $H^{2}(G, T)$ with a 2-cocycle $\varphi$ in $Z^{2}(G, T)$. Since the image of $\varphi$ is a compact subset of the discrete toplogical group $T$, there exists a positive integer $m$ such that the image of $\varphi$ is contained in the subgroup $C_{m}=(1 / m) \mathbf{Z} / \mathbf{Z}$ of $T$. Then $\varphi$ belongs to $Z^{2}\left(G, C_{m}\right)$ and determines a central extension of profinite groups

$$
1 \longrightarrow C_{m} \longrightarrow H \stackrel{\pi}{\longrightarrow} G \longrightarrow 1 \text {. }
$$

Since $\rho: F \rightarrow G$ gives a presentation of $G$ with a free profinite group $F$, there exists a homomorphism $\eta$ of $F$ to $H$ such that $\pi \circ \eta=\rho$. Then $\eta(R)$ is contained in (the image of) $C_{m}$. Take a continuous cross-section $\sigma: G$ $\rightarrow F$ of $\rho$ (cf. [Ko], Satz 1.16, p. 8, or [Sh], Theorem 3, p. 10), and put $\xi=\eta \circ \sigma$. Then we have a 2-cocycle $\psi \in Z^{2}\left(G, C_{m}\right)$ defined by

$$
\psi(x, y)=\xi(x y)^{-1} \xi(x) \xi(y), \quad x, y \in G,
$$

because $\xi$ is a cross-section of $\pi: H \rightarrow G$. By the choice of $H, \psi$ is cohomologous to $\varphi$. Therefore it belongs to the class $(\varphi)$ in $H^{2}(G, T)$. Now let $\chi$ be the element of $\operatorname{Hom}(R, T)$ obtained by restricting $\eta$ to $R$ (combined with the inclusion $C_{m} \rightleftarrows T$ ). Then we have

$$
\begin{aligned}
\psi(x, y) & =\eta(\sigma(x y))^{-1} \eta(\sigma(x)) \eta(\sigma(y)) \\
& =\eta\left(\sigma(x y)^{-1} \sigma(x) \sigma(y)\right) \\
& =\chi\left(\sigma(x y)^{-1} \sigma(x) \sigma(y)\right)
\end{aligned}
$$

for $x, y \in G$. Since $\sigma$ is a cross-section of $\rho$, this shows that $\tau(\chi)=(\psi)$ $=(\varphi)$, which proves that $\tau$ is surjective.

If we take another presentation $G=F^{\prime} / R^{\prime}$ with a free profinite group $F^{\prime}$, then we also have an isomorphism

$$
\tau^{\prime}: \operatorname{Hom}\left(R^{\prime} \cap\left[F^{\prime}, F^{\prime}\right] /\left[R^{\prime}, F^{\prime}\right], T\right) \stackrel{\sim}{\longrightarrow} H^{2}(G, T)
$$

by the theorem. It is easy to see, however, that there exists a canonical homomorphism of $F$ to $F^{\prime}$ which induces a homomorphism

$$
\theta: R \cap[F, F] /[R, F] \longrightarrow R^{\prime} \cap\left[F^{\prime}, F^{\prime}\right] /\left[R, F^{\prime}\right] .
$$

The dual $\hat{\theta}$ satisfies the condition $\tau^{\prime}=\tau \circ \hat{\theta}$ and is an isomorphism. Therefore $\theta$ is an isomorphism of compact abelian groups. This observation allows us to define

$$
\mathfrak{M}(G):=R \cap[F, F] /[R, F]
$$


Then the statement of the theorem is dualized as follows:

Theorem $1^{\prime}$. For a profinite group $G$, the Schur multiplier $H^{2}(G, T)$ is canonically dual to $\mathfrak{M}(G)$.

\section{§3. The structure of $\mathfrak{M}(G)$ as a profinite group}

Let $G$ be a profinite group and $N$ be its closed normal subgroup. Then a homomorphism

$$
\gamma=\gamma_{N}^{G}: \mathfrak{M}(G) \longrightarrow \mathfrak{M}(G / N)
$$

is canonically determined by the definition. The cokernel of $\gamma$ is also determined; the following sequence is exact (cf. [B-E], Theorem 1.1, p. 101):

$$
\mathfrak{M}(G) \stackrel{r}{\longrightarrow} \mathfrak{M}(G / N) \longrightarrow N \cap[G, G] /[N, G] \longrightarrow 1 .
$$

On the other hand, we have the inflation map

$$
\lambda=\lambda_{N}^{G}: H^{2}(G / N, T) \longrightarrow H^{2}(G, T) ;
$$

its kernel can be determined by the Hochschild-Serre exact sequence

$\left(1^{\prime}\right) \quad 1 \longrightarrow \operatorname{Hom}(N \cap[G, G] /[N, G], T) \longrightarrow H^{2}(G / N, T) \stackrel{\lambda}{\longrightarrow} H^{2}(G, T)$.

Using Theorem $1^{\prime}$ we see

Proposition 2. The exact sequence $\left(1^{\prime}\right)$ is dual to (1).

Altogether this shows

Proposition 3. Let the notation and the assumptions be as above. Denote the dual map of $\gamma_{N}^{G}$ by $\hat{\gamma}_{N}^{G}$, and let $\tau_{G}$ and $\tau_{G / N}$ be the isomorphisms given in Theorem 1 for $G$ and for $G / N$, respectively. Then we have a commutative diagram

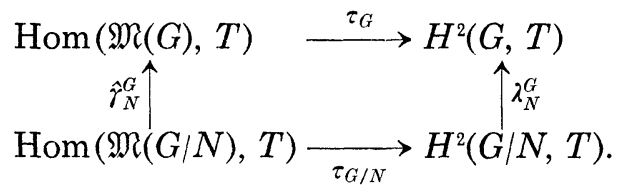

Now let $\mathfrak{H}$ be the family of all open normal subgroups of $G$. For $U, V \in \mathfrak{H}, U \supset V$, we have a homomorphism

$$
\gamma_{U, V}: \mathfrak{M}(G / V) \longrightarrow \mathfrak{M}(G / U)
$$

together with 


$$
\gamma_{U}: \mathfrak{M}(G) \longrightarrow \mathfrak{M}(G / U)
$$

and

$$
\gamma_{V}: \mathfrak{M}(G) \longrightarrow \mathfrak{M}(G / V) .
$$

From the definition we see

$$
\gamma_{U}=\gamma_{U, V} \circ \gamma_{V}
$$

and

$$
\gamma_{U, W}=\gamma_{U, V} \circ \gamma_{V, W}
$$

for $U, V, W \in \mathfrak{H}, U \supset V \supset W$. Therefore we have a projective system of finite abelian groups

$$
\left\{\mathfrak{M}(G / U), \gamma_{U, V} \mid U, V \in \mathfrak{H}, U \supset V\right\} .
$$

We have also an inductive system of Schur multipliers

$$
\left\{H^{2}(G / U, T), \lambda_{V, U} \mid U, V \in \mathfrak{H}, U \supset V\right\}
$$

where $\lambda_{V, U}$ is the inflation map

$$
\lambda_{V, U}: H^{2}(G / U, T) \longrightarrow H^{2}(G / V, T),
$$

and also a system of homomorphisms

$$
\lambda_{U}: H^{2}(G / U, T) \longrightarrow H^{2}(G, T), \quad U \in \mathfrak{H} .
$$

Since the action of $G$ on $T$ is trivial, we have

$$
H^{2}(G, T)=\varliminf_{U} H^{2}(G / U, T),
$$

(cf. [Sh], Corollary 1, p. 26); and then

$$
\text { Proposition 4. } \quad \mathfrak{M}(G)=\lim _{U \in \mathfrak{U}} \mathfrak{M}(G / U) .
$$

Proof. Put $H=\varliminf_{\mathfrak{M}} \mathfrak{M}(G / U)$. Then by (3) and the universal property of $H$, we have a continuous homomorphism $\varphi: \mathfrak{M}(G) \rightarrow H$. Because of (2), the two systems (4) and (5) are dual to each other. By (6), therefore, $H^{2}(G, T)$ is the dual group of $H$. Then by Theorem $1^{\prime}$ and (2), we conclude that $\varphi$ is an isomorphism.

\section{§4. The structure of $\operatorname{Gal}\left(k^{(c+1)} / k^{(c)}\right)$}

Let us go back to Galois groups of nilpotent extensions of an algebraic number field $k$. An open normal subgroup $U$ of $G^{c}$ corresponds to a 
finite normal subextension $K / k$ of $k^{(c)} / k$ in such a way that $U=\operatorname{Gal}\left(k^{(c)} / K\right)$. Therefore we obtain from Theorem $1^{\prime}$ and Proposition 4

Theorem 2. (i) For each $c \geqq 1$ there is a canonical isomorphism

$$
\iota^{c}: \mathfrak{M}\left(G^{c}\right) \stackrel{\sim}{\longrightarrow} N^{c+1}=\mathrm{Gal}\left(k^{(c+1)} / k^{(c)}\right) .
$$

(ii) $\mathfrak{M}\left(G^{c}\right)$ is determined by finite normal subextensions $K / k$ of $k^{(c)} / k$ as

$$
\mathfrak{M}\left(G^{c}\right)=\varliminf_{K}^{\lim } \mathfrak{M}(\mathrm{Gal}(K / k)) \text {. }
$$

For a finite normal subextension $K / k$ of $k^{(c)} / k$ with $U=\operatorname{Gal}\left(k^{(c)} / K\right)$ denote by

$$
\gamma_{K}=\gamma_{U}: \mathfrak{M}\left(G^{c}\right) \longrightarrow \mathfrak{M}(\operatorname{Gal}(K / k))
$$

the natural homomorphism determined by (ii) of the theorem. We denote the maximal central extension of $K / k$ in $\overline{\mathbf{Q}}$ by $M C(K / k)$. This is a subfield of $k^{(c+1)}$ because $K / k$ is a subextension of $k^{(c)} / k$.

In Section 6 we shall prove

THEOREM 3. For each finite normal subextension $K / k$ of $k^{(c)} / k$, there exists a canonical isomorphism

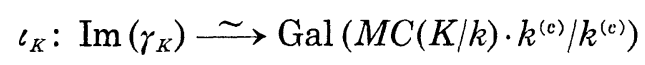

such that the following diagram is commutative:

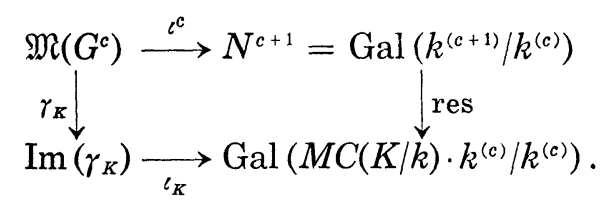

\section{§5. Base-change for abundant central extensions}

In this section let $K / k$ be a Galois extension of algebraic number fields of finite degree. Put $\mathfrak{g}=\mathrm{Gal}(K / k)$. The maximal central extension $M C(K / k)$ of $K / k$ contains $K \cdot k^{\text {ab }}$. There exists a canonical isomorphism

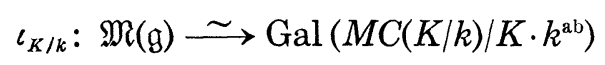

(see e.g. [Mi]). Since $M C(K / k)$ is a finite extension of $K \cdot k^{\text {ab }}$, there is a finite central extension $L$ of $K / k$ such that $M C(K / k)$ is equal to the composite field $L \cdot k^{\text {ab }}$. Such an $L$ is called an abundant central extension 
of $K / k$. Put $L^{*}=L \cap K \cdot k^{\mathrm{ab}}$. Then the isomorphism $\iota_{K / k}$ induces an isomorphism of $\mathfrak{M}(\mathfrak{g})$ onto $\mathrm{Gal}\left(L / L^{*}\right)$ if $L$ is abundant. Suppose that another finite Galois extension $K_{1} / k, K_{1} \supset K$, is given, and put $G=\operatorname{Gal}\left(K_{1} / k\right)$ and $N=\operatorname{Gal}\left(K_{1} / K\right)$. Then $\mathfrak{g}=G / N$. The homomorphism $\gamma$ of (1) in Section 3 gives a basic relation between $\mathfrak{M}(G)$ and $\mathfrak{M}(\mathfrak{g})$. Let $\hat{K}_{1}$ be the maximal central extension of $K / k$ in $K_{1}$, i.e. $\hat{K}_{1}=K_{1} \cap M C(K / k)$, and let $K_{1}^{*}$ be the genus field, i.e. $K_{1}^{*}=K_{1} \cap K \cdot k^{\text {ab }}$. Then by the definition we see that $\operatorname{Gal}\left(\hat{K}_{1} / K_{1}^{*}\right)$ is isomorphic to $N \cap[G, G] /[N, G]$, the third term of the exact sequence (1). Now let us denote the composite field $L \cdot K_{1}$ by $L_{1}$, and put $L_{1}^{* *}=L_{1} \cap K_{1} \cdot k^{\mathrm{ab}}$. Since $L_{1}$ is a central extension of $K_{1} / k$, $\operatorname{Gal}\left(L_{1} / L_{1}^{* *}\right)$ is a homomorphic image of $\mathfrak{M}(G)$ under the map induced by the canonical homomorphism $\iota_{K_{1} / k}$ for the Galois extension $K_{1} / k$.

THEOREM 4. Let the notation and the assumptions be as above. Then $L_{1}$ is a central extension of $K_{1} / k$ with the following properties:

(i ) $\mathrm{Gal}\left(L_{1} / L_{1}^{* *}\right)$ is canonically isomorphic to $\mathrm{Gal}\left(L / L \cap L_{1}^{* *}\right)$;

(ii) $\mathrm{Gal}\left(L \cap L_{1}^{* *} / L^{*}\right)$ is canonically isomorphic to $\mathrm{GaJ}\left(\hat{K}_{1} / K_{1}^{*}\right)$;

(iii) We have a commutative diagram with exact rows

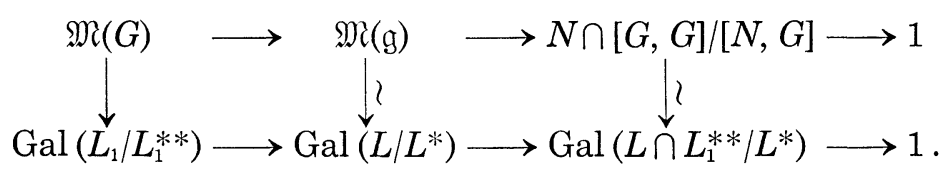

Proof. Put $\mathfrak{S}=\operatorname{Gal}\left(L_{1} / k\right)$ and $\mathfrak{N}=\operatorname{Gal}\left(L_{1} / K\right) ; \mathfrak{N}$ is a normal subgroup with quotient $\mathfrak{g}=\mathfrak{S} / \mathfrak{R}$. Put $\mathfrak{A}=\operatorname{Gal}\left(L_{1} / K_{1}\right)$ and $\mathfrak{B}=\operatorname{Gal}\left(L_{1} / L\right)$; then $\operatorname{Gal}\left(L_{1} / L \cap K_{1}\right)$ is a direct product $\mathfrak{B} \times \mathfrak{U}$ because $L_{1}=L \cdot K_{1}$. Let $\hat{L}_{1}$ be the subfield of $L_{1}$ determined by the condition $\operatorname{Gal}\left(L_{1} / \hat{L}_{1}\right)=[\Re, \mathfrak{S}]$; this is the maximal central extension of $K / k$ in $L_{1}$, i.e. $\hat{L}_{1}=L_{1} \cap M C(K / k)$; it contains $L$. Therefore we have $[\mathfrak{N}, \mathfrak{S}] \subset \mathfrak{B}$. Since $\mathfrak{A}$ is a normal subgroup of $\mathfrak{S}$ contained in $\mathfrak{R}$, the commutator $[\mathfrak{U}, \mathfrak{S}]$ is contained in $\mathfrak{A} \cap[\mathfrak{N}, \mathfrak{S}]$; hence we have $[\mathfrak{U}, \mathfrak{S}]=1$ because $\mathfrak{A} \cap \mathfrak{B}=1$; this shows that $\mathfrak{A}$ lies in the center of $\mathfrak{S}$, which means that $L_{1}$ is a central extension of $K_{1} / k$.

Now let us see (i). We have $\operatorname{Gal}\left(L_{1} / L_{1}^{* *}\right)=\mathfrak{U} \cap\left[\mathfrak{S}_{\mathcal{E}}, \mathfrak{S}_{\mathrm{C}}\right]$ because $L_{1}^{* *}=$ $L_{1} \cap K_{1} \cdot k^{\mathrm{ab}}=K_{1} \cdot\left(L_{1} \cap k^{\mathrm{ab}}\right)$ and $\operatorname{Gal}\left(L_{1} / L_{1} \cap k^{\mathrm{ab}}\right)=[\mathfrak{S}, \mathfrak{S}]$. Therefore Gal $\left(L_{1} /\right.$ $\left.L \cap L_{1}^{* *}\right)=\mathfrak{B} \times \operatorname{Gal}\left(L_{1} / L_{1}^{* *}\right)$ because $\operatorname{Gal}\left(L_{1} / L \cap K_{1}\right)=\mathfrak{B} \times \mathfrak{A}$. Hence it is obvious that the projection of $\operatorname{Gal}\left(L_{1} / L \cap L_{1}^{* *}\right)$ onto $\operatorname{Gal}\left(L / L \cap L_{1}^{* *}\right)$ maps $\operatorname{Gal}\left(L_{1} / L_{1}^{* *}\right)$ isomorphically onto $\operatorname{Gal}\left(L / L \cap L_{1}^{* *}\right)$. This proves (i). Note that $\operatorname{Gal}\left(L_{1} / L_{1}^{* *}\right)$ is a homomorphic image of $\mathfrak{M}(G)$ because $L_{1}^{* *}$ is the genus field of the central extension $L_{1}$ of $K_{1} / k$. Put $L_{1}^{*}=L_{1} \cap K \cdot k^{\text {ab }}$; this 


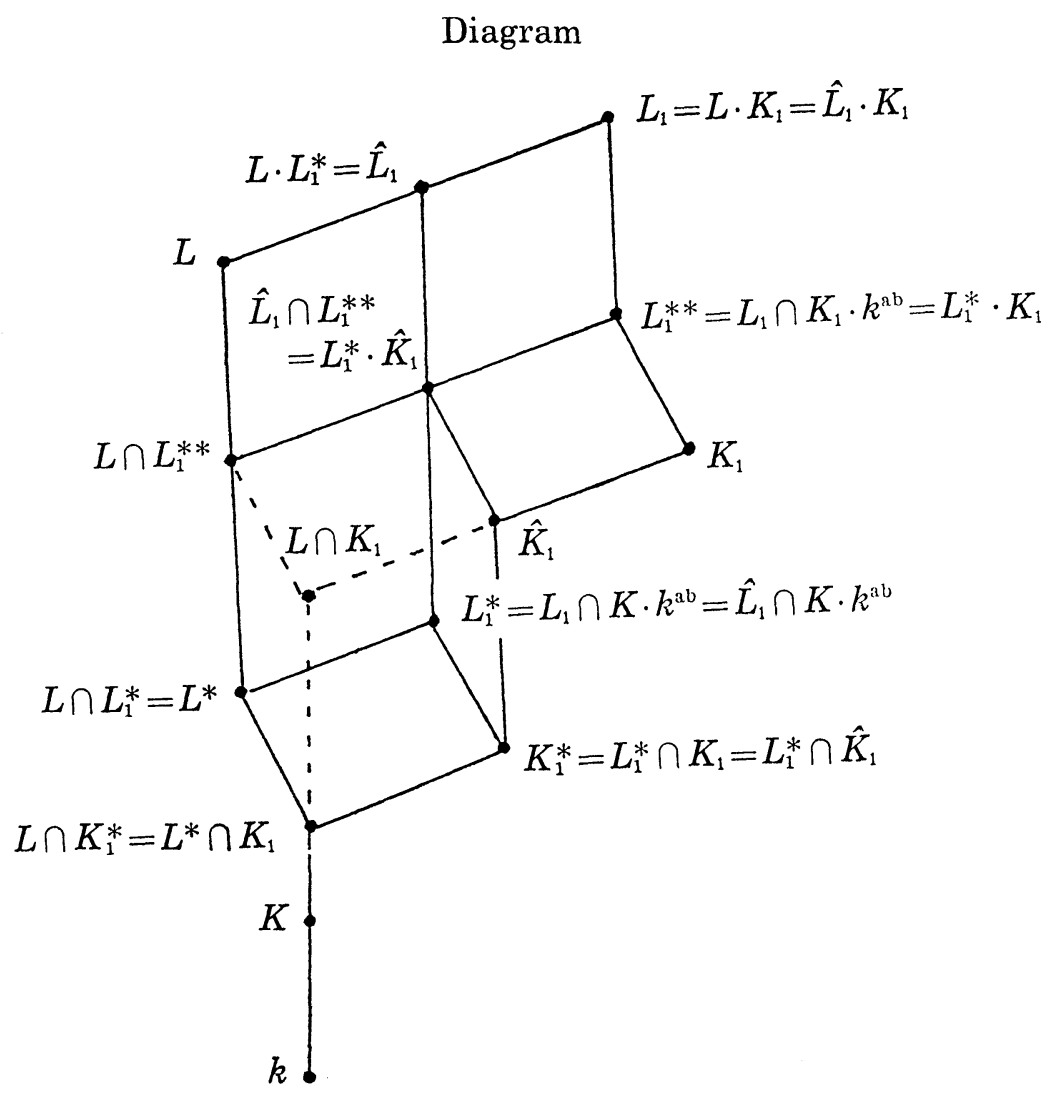

is the genus field of the central extension $\hat{L}_{1}=L_{1} \cap M C(K / k)$ of $K / k$. Since $L_{1}^{*}=K \cdot\left(L_{1} \cap k^{\text {ab }}\right)$, we have $\operatorname{Gal}\left(L_{1} / L_{1}^{*}\right)=\mathfrak{R} \cap\left[\mathfrak{S}_{2}, \mathfrak{g}\right]$. By the assumption, $L$ is an abundant central extension of $K / k$. Therefore $\hat{L}_{1}$ is contained in $L \cdot k^{\mathrm{ab}}$ and hence equal to $L \cdot L_{1}^{*}$. Then $\operatorname{Gal}\left(\hat{L}_{1} / L_{1}^{*}\right)$ is naturally isomorphic to $\operatorname{Gal}\left(L / L^{*}\right)$ because $L \cap L_{1}^{*}=L \cap K \cdot k^{\text {ab }}=L^{*}$. Under this isomorphism the intermediate field $L \cap L_{1}^{* *}$ of $L / L^{*}$ corresponds to $\left(L \cap L_{1}^{* *}\right) \cdot L_{1}^{*}$. Furthermore, we also have natural isomorphisms

$$
\operatorname{Gal}\left(\hat{L}_{1} / L\right) \cong \operatorname{Gal}\left(\left(L \cap L_{1}^{* *}\right) \cdot L_{1}^{*} / L \cap L_{1}^{* *}\right) \cong \operatorname{Gal}\left(L_{1}^{*} / L^{*}\right) .
$$

Since there is a natural isomorphism of $\operatorname{Gal}\left(L_{1} / L\right)$ onto $\operatorname{Gal}\left(L_{1}^{* *} / L \cap L_{1}^{* *}\right)$, we conclude that the intermediate field $\left(L \cap L_{1}^{* *}\right) \cdot L_{1}^{*}$ coincides with $\hat{L}_{1} \cap L_{1}^{* *}$. Therefore $\operatorname{Gal}\left(L \cap L_{1}^{* *} / L^{*}\right)$ is naturally isomorphic to $\operatorname{Gal}\left(\hat{L}_{1} \cap L_{1}^{* *} / L_{1}^{*}\right)$. Next let us look at the extension $L_{1}^{* *} / K_{1}^{*}$. By definition we easily see $L_{1}^{*} \cap K_{1}=K_{1}^{*}$. Hence $\operatorname{Gal}\left(L_{1} / K_{1}^{*}\right)=\left(\mathfrak{R} \cap\left[\mathfrak{S}_{\mathrm{E}}, \mathfrak{S}\right]\right) \cdot \mathscr{R}$ because $\operatorname{Gal}\left(L_{1} / L_{1}^{*}\right)=$ $\mathfrak{R} \cap\left[\mathfrak{S}_{\mathfrak{B}}, \mathfrak{S}\right]$ and $\operatorname{Gal}\left(L_{1} / K_{1}\right)=\mathfrak{A}$. Therefore we obtain 


$$
\begin{aligned}
\operatorname{Gal}\left(L_{1}^{*} / K_{1}^{*}\right) & =(\mathfrak{N} \cap[\mathfrak{S}, \mathfrak{S}]) \cdot \mathfrak{U} /\left(\mathfrak{N} \cap\left[\mathfrak{S}, \mathfrak{S}_{\mathrm{C}}\right]\right) \\
& \cong \mathfrak{A} / \mathfrak{U} \cap(\mathfrak{N} \cap[\mathfrak{S}, \mathfrak{S}]) \\
& =\mathfrak{U} / \mathfrak{A} \cap\left[\mathfrak{S}_{\mathfrak{S}}, \mathfrak{S}\right] \\
& =\operatorname{Gal}\left(L_{1}^{* *} / K_{1}\right)
\end{aligned}
$$

In particular we have $\left[L_{1}^{* *}: K_{1}\right]=\left[L_{1}^{*}: K_{1}^{*}\right]$. It is clear that $L_{1}^{* *}$ contains $L_{1}^{*} \cdot K_{1}$. Since $\left[L_{1}^{*} \cdot K_{1}: K_{1}\right]=\left[L_{1}^{*}: L_{1}^{*} \cap K_{1}\right]=\left[L_{1}^{*}: K_{1}^{*}\right]=\left[L_{1}^{* *}: K_{1}\right]$, we conclude $L_{1}^{* *}=L_{1}^{*} \cdot K_{1}$. This implies that $\operatorname{Gal}\left(\hat{L}_{1} \cap L_{1}^{* *} / L_{1}^{*}\right)$ is naturally isomorphic to $\mathrm{Gal}\left(\hat{K}_{1} / K_{1}^{*}\right)$ because $\hat{K}_{1}=\left(\hat{L}_{1} \cap L_{1}^{* *}\right) \cap K_{1}$. Combining this with the result obtained above, we have shown that Gal $\left(L \cap L_{1}^{* *} / L^{*}\right)$ is naturally isomorphic to $\mathrm{Gal}\left(\hat{K}_{1} / K_{1}^{*}\right)$ as is claimed in (ii).

The last Galois group is isomorphic to

$$
\operatorname{Gal}\left(K_{1} / K_{1}^{*}\right) / \operatorname{Gal}\left(K_{1} / \hat{K}_{1}\right)=N \cap[G, G] /[N, G] .
$$

Since all the isomorphisms of Galois groups discussed above are natural and group-theoretic, (iii) is also clear.

\section{§6. The proof of Theorem 3}

We use the same notation as in Section 4 . Let $K / k$ be a finite normal subextension of $k^{(c)} / k, c \geqq 1$; its Galois group is denoted by $\mathrm{g}=$ $\operatorname{Gal}(K / k) ; U=\operatorname{Gal}\left(k^{(c)} / K\right)$ is an open normal subgroup of $G^{c}$. We fix an abundant central extension $L$ of $K / k ; M C(K / k) \cdot k^{(c)}$ is equal to $L \cdot k^{(c)}$. Put $K_{0}=L \cap k^{(c)}$; then $\mathrm{Gal}\left(L / K_{0}\right)$ is canonically isomorphic to $\mathrm{Gal}\left(M C(K / k) \cdot k^{(c)}\right.$ $\left.\mid k^{(c)}\right)$. Put $\tilde{U}=\operatorname{Gal}\left(k^{(c)} / K_{0}\right), G=\operatorname{Gal}\left(K_{0} / k\right)$ and $N=\operatorname{Gal}\left(K_{0} / K\right)$. We use Theorem 4 for $L / K / k$ and $K_{1}=K_{0}$; in this case we have $L \supset K_{1} \supset L^{*}$; $N$ lies in the center of $G$, and $[N, G]=1$; therefore $K_{1}=\hat{K}_{1}, K_{1}^{*}=L^{*}$; moreover we have $L_{1}=L, L_{1}^{*}=L^{*}$ and $L_{1}^{* *}=K_{1}=L \cap L_{1}^{* *}$; hence the image of the homomorphism

$$
\gamma_{N}^{G}: \mathfrak{M}(G) \longrightarrow \mathfrak{M}(\mathfrak{g})
$$

is mapped isomorphically onto $\mathrm{Gal}\left(L / K_{0}\right)$ by the isomorphism

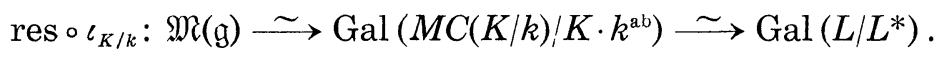

Let us express this using $U$ and $\tilde{U}$; we have $\mathfrak{M}(G)=\mathfrak{M}\left(G^{c} / \tilde{U}\right), \mathfrak{M}(\mathfrak{g})=$ $\mathfrak{M}\left(G^{c} / U\right)$ and $\gamma_{N}^{G}=\gamma_{U, \tilde{U}}$ in the notation of Section 3; furthermore, the image of $\gamma_{U, \tilde{U}}, \operatorname{Im}\left(\gamma_{U, \tilde{U}}\right)$, is mapped isomorphically onto the subgroup of $\operatorname{Gal}\left(M C(K / k) / K \cdot k^{\text {ab }}\right)$ by $\iota_{K / k}$ which is canonically isomorphic to $\mathrm{Gal}\left(L / K_{0}\right)$ and hence also to $\operatorname{Gal}\left(M C(K / k) \cdot k^{(c)} / k^{(c)}\right)$. Next let $V$ be an open normal 
subgroup of $G^{c}$ contained in $U$; put $\tilde{V}=V \cap \tilde{U}$; then we have

$$
\gamma_{U, \tilde{V}}=\gamma_{U, V} \circ \gamma_{V, \tilde{V}}=\gamma_{U, \tilde{U}} \circ \gamma_{\tilde{U}, \tilde{V}}
$$

hence, in order to show $\operatorname{Im} \gamma_{U}=\operatorname{Im}\left(\gamma_{U}, \tilde{U}\right)$ for $\gamma_{U}\left(=\gamma_{K}\right)=\lim _{V \subset U} \gamma_{U, V}$, it is sufficient to prove that $\operatorname{Im}\left(\gamma_{U}, \tilde{v}\right)$ in $\mathfrak{M}(\mathfrak{g})$ is mapped isomorphically onto $\operatorname{Gal}\left(L / K_{0}\right)$ by res $\circ \iota_{K / k}$. This time we use Theorem 4 for $L / K / k$ and the extension $K_{1} / k$ determined by the condition $\operatorname{Gal}\left(K_{1} / k\right)=G^{c} / \tilde{V}$. We have $K_{1} \supset K_{0}$ by the choice of $V$; since $K_{1}$ is a subfield of $k^{(c)}$, we also have $L \cap K_{1}=K_{0}$ and hence $L^{*} \subset L \cap K_{1}$. For $c \geqq 1, L_{1}^{* *}=L_{1}^{*} \cdot K_{1}$ is contained in $k^{(c)}$ because $k^{(c)} \supset K \cdot k^{\text {ab }}$ and $L_{1}^{*}=L_{1} \cap K \cdot k^{\text {ab }}$; therefore $L \cap L_{1}^{* *} \subset L \cap k^{(c)}$ $=K_{0}$; conversely, it is clear that $L \cap L_{1}^{* *} \supset K_{0}=L \cap K_{1}$; thus we have $L \cap L_{1}^{* *}=K_{0}$. It now follows from Theorem 4 that the image of the homomorphism

$$
\gamma_{U, \tilde{v}}: \mathfrak{M}\left(G^{c} / \tilde{V}\right) \longrightarrow \mathfrak{M}\left(G^{c} / U\right)
$$

is isomorphically mapped onto $\operatorname{Gal}\left(L / K_{0}\right)$ by $\operatorname{res} \circ \iota_{K / k}$. This proves that $\operatorname{Im} \gamma_{U}, \gamma_{U}=\gamma_{K}$, coincides with $\operatorname{Im}\left(\gamma_{U}, \tilde{U}\right)$ and also that there exists a canonical isomorphism

$$
\iota_{K}: \operatorname{Im} \gamma_{K} \longrightarrow \operatorname{Gal}\left(M C(K / k) \cdot k^{(c)} / k^{(c)}\right) .
$$

The rest of Theorem 3 will easily be seen in a straightforward way by dualizing the diagram of Theorem 4.

\section{§ 7. The canonical 2-cohomology classes}

We fix an algebraic number field $k$ of finite degree. Let $K$ be a finite Galois extension of $k, K_{\mathbf{A}}^{\times}$be the idele group of $K, K_{\infty+}^{\times}$be the connected component of the identity element of the Archimedian part of $K_{\mathrm{A}}^{\times}$and $K^{\sharp}$ be the closure of $K^{\times} \cdot K_{\infty+}^{\times}$in $K_{\mathbf{A}}^{\times}$. We have the Artin map of $K$,

$$
\alpha_{K}: K_{\mathrm{A}}^{\times} / K^{\#} \longrightarrow \operatorname{Gal}\left(K^{\mathrm{ab}} / K\right),
$$

which is a topological isomorphism, and the natural exact sequence

$$
E(K / k): \quad 1 \longrightarrow K_{\mathbf{A}}^{\times} / K^{\#} \longrightarrow \operatorname{Gal}\left(K^{\mathrm{ab}} / k\right) \longrightarrow \operatorname{Gal}(K / k) \longrightarrow 1 .
$$

The structure of $\mathrm{Gal}\left(K^{\mathrm{ab}} / k\right)$ is then determined by the canonical 2-cohomology class $\bar{\xi}_{K / k}$ of $\mathrm{Gal}(K / k)$ with values in $K_{\mathrm{A}}^{\times} / K^{\#}$. More specifically, the cohomology group $H^{2}\left(\mathrm{Gal}(K / k), K_{\mathrm{A}}^{\times} / K^{\#)}\right.$ is a cyclic group generated by $\bar{\xi}_{K / k}$; its order is either $\frac{1}{2} \cdot[K: k]$ if there exists a ramified real Archimedian prime in $K / k$ or $[K: k]$ otherwise (cf. Katayama [Kt] and also 
Iyanaga [Iy]). In this sense $\operatorname{Gal}\left(K^{\mathrm{ab}} / k\right)$ is determined by $K / k$.

Let $F / k, F \supset K$, be another finite Galois extension, $F_{\mathbf{A}}^{\times} / F^{\#}$ be as above for $F$ and

$$
N_{F / K}: F_{\mathbf{A}}^{\times} / F^{\#} \longrightarrow K_{\mathbf{A}}^{\times} / K^{\#}
$$

be the norm map of $F / K$. Then we have a commutative diagram

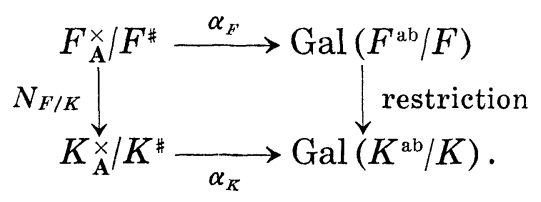

Therefore $N_{F / K}$ and the homomorphisms defined by restricting automorphisms of $F^{\text {ab }}$ or of $F$ to $K^{\text {ab }}$ or to $K$, respectively, give a homomorphism of the exact sequence $E(F / k)$ to $E(K / k)$.

Now suppose that an infinite Galois extension $\tilde{k} / k$ is given. Put $G=\operatorname{Gal}(\tilde{k} / k)$. If we make $K / k$ run over all finite Galois subextensions of $\tilde{k} / k$, we have projective systems $\left\{K_{\mathbf{A}}^{\times} / K^{\#}, N_{F / K}\right\},\left\{\operatorname{Gal}\left(K^{\text {ab }} / K\right)\right\},\left\{\operatorname{Gal}\left(K^{\text {ab }} / k\right)\right\}$, $\{\operatorname{Gal}(K / k)\}$ and $\{E(K / k)\}$, and also

$$
\begin{aligned}
& \operatorname{Gal}\left(\tilde{k}^{\mathrm{ab}} / k\right)=\varliminf_{K} \operatorname{Gal}\left(K^{\mathrm{ab}} / k\right), \\
& \operatorname{Gal}\left(\tilde{k}^{\mathrm{ab}} / \tilde{k}\right)=\varliminf_{K} \operatorname{Gal}\left(K^{\mathrm{ab}} / K\right), \\
& G=\operatorname{Gal}(\tilde{k} / k)=\varliminf_{K} \operatorname{Gal}(K / k) .
\end{aligned}
$$

We put

$$
\mathfrak{X}(\tilde{k})=\varliminf_{\frac{1}{K}} K_{\mathbf{A}}^{\times} / K^{\sharp} .
$$

It is clear that $\mathfrak{Q}(\tilde{k})$ depends only on $\tilde{k}$. Each $K_{\mathrm{A}}^{\times} / K^{\#}$ is naturally considered as a $G$-module. Therefore $\mathfrak{A}(\tilde{k})$ has a $G$-module structure. Through inner automorphisms of $\operatorname{Gal}\left(\tilde{k}^{\mathrm{ab}} / k\right), \operatorname{Gal}\left(\tilde{k}^{\mathrm{ab}} / \tilde{k}\right)$ becomes a $G$-module.

Proposition 5. Let the notation and the assumptions be as above. The Artin maps $\alpha_{K}$ for finite Galois subextensions $K / k$ of $\tilde{k} / k$ give a $G$ isomorphism

$$
\alpha_{\tilde{k}}=\lim _{K} \alpha_{K}: \mathfrak{A}(\tilde{k}) \longrightarrow \operatorname{Gal}\left(\tilde{k}^{\mathrm{a} b} / \tilde{k}\right) .
$$

The exact sequence

$$
E(\tilde{k} / k): \quad 1 \longrightarrow \mathfrak{A}(\tilde{k}) \longrightarrow \operatorname{Gal}\left(\tilde{k}^{\mathrm{ab}} / k\right) \longrightarrow \operatorname{Gal}(\tilde{k} / k) \longrightarrow 1
$$


determined naturally by $\alpha_{\tilde{k}}$ is the projective limit of $\{E(K / k)\}$. Therefore the canonical classes $\bar{\xi}_{K / k}$ determine the canonical 2-cohomology class $\bar{\xi}_{\tilde{k} / k}$ in $H^{2}(G, \mathfrak{Y}(\tilde{k}))$ which gives the extension $\operatorname{Gal}\left(\tilde{k}^{\mathrm{ab}} / k\right)$ of $\mathrm{Gal}(\tilde{k} / k)$ by $\mathfrak{A}(\tilde{k})$.

The proof is almost obvious because lim is an exact functor in the category of compact groups (e.g. [E-S], Theorem 5.6, p. 226, or [Ko], Satz 1.9 , p. 6).

Corollary. Let $M C(\tilde{k} / k)$ be the maximal central extension of $\tilde{k} / k$. Then we have

$$
\operatorname{Gal}(M C(\tilde{k} / k) / \tilde{k})=\mathfrak{2}(\tilde{k}) / \mathfrak{A}(\tilde{k})^{\Delta G}
$$

where

$$
\mathfrak{U}(\tilde{k})^{\Delta G}=\left\langle x^{1-\sigma} \mid x \in \mathfrak{U}(\tilde{k}), \sigma \in G\right\rangle
$$

(the right-hand side means the topologically generated closed subgroup).

Let us apply these results to the case where $\tilde{k} / k$ is the nilpotent extension $k^{(c)} / k, c \geqq 1$, and $G=G^{c}$. Then since $k^{(c+1)}=M C\left(k^{(c)} / k\right)$ we obtain from Theorem 2 the following result:

TheOREM 5. (i) For each $c \geqq 1$, there exists a surjective homomorphism $\alpha^{c}: \mathfrak{A}\left(k^{(c)}\right) \rightarrow \mathfrak{M}\left(G^{c}\right)$ with $\operatorname{Ker} \alpha^{c}=\mathfrak{A}\left(k^{(c)}\right)^{\Delta G c}$ such that the homomorphism

$$
\iota^{c} \circ \alpha^{c}: \mathfrak{A}\left(k^{(c)}\right) \longrightarrow N^{c+1}=\operatorname{Gal}\left(k^{(c+1)} / k^{(c)}\right)
$$

coincides with the homomorphism induced naturally from the Artin map

$$
\alpha_{k^{(c)}}: \mathfrak{A}\left(k^{(c)}\right) \longrightarrow \operatorname{Gal}\left(k^{(c), a b} / k^{(c)}\right) .
$$

(ii) The group extension

$$
1 \longrightarrow \mathfrak{M}\left(G^{c}\right) \stackrel{\iota^{c}}{\longrightarrow} G^{c+1} \longrightarrow G^{c} \longrightarrow 1
$$

determined by $\iota^{c}: \mathfrak{M}\left(G^{c}\right) \rightarrow \operatorname{Gal}\left(k^{(c+1)} / k^{(c)}\right)$ corresponds to the image of the canonical class $\bar{\xi}_{k^{\prime}(c) / k}$ under the induced homomorphism

$$
\left(\alpha^{c}\right)^{*}: H^{2}\left(G^{c}, \mathfrak{A}\left(k^{(c)}\right)\right) \longrightarrow H^{2}\left(G^{c}, \mathfrak{M}\left(G^{c}\right)\right) .
$$

Remark. For $g$ and $h \in G^{c}$, take $\tilde{g}$ and $\tilde{h} \in G^{c+1}$ over $g$ and $h$, respectively. Then the commutator $[\tilde{g}, \tilde{h}]$ depends only on $g$ and $h$ because the group extension of Theorem 5, (ii), is central. Hence from the definition we obtain an epimorphism 


$$
\varphi^{c}: N^{c} \otimes G^{c} \longrightarrow \mathfrak{M}\left(G^{c}\right)
$$

such that

$$
\iota^{c}(\varphi(n, g))=[\tilde{n}, \tilde{g}]
$$

for $n \in N^{c}$ and $g \in G^{c}$. Let $f$ be a 2-cocycle which belongs to the cohomology class $\left(\alpha^{c}\right)^{*}\left(\bar{\xi}_{k^{(c)} / k}\right) \in H^{2}\left(G^{c}, \mathfrak{M}\left(G^{c}\right)\right)$. Then it is easily seen that for $n \in N^{c}$ and $g \in G^{c}$ we have

$$
\varphi(n, g)=f(n, g) \cdot f(g, n)^{-1}
$$

because $N^{c}$ lies in the center of $G^{c}$. For a finite abelian extension $K / k$ with $G=N=\operatorname{Gal}(K / k)$, a detailed analysis of the analogous map is given by Furuta [Fu].

Remark. The main body of our present results relies on two facts; one of them is that Schur multipliers of profinite groups and their duals have good functorial properties; the other is a result of Tate, $\left.H^{2}(\&), \mathbf{Q} / \mathbf{Z}\right)$ $=0$ for $\mathbb{B S}=\operatorname{Gal}(\overline{\mathbf{Q}} / k)$, from which we not only deduce Proposition 1 but also the existence of abundant central extensions of a finite Galois subextension of $\overline{\mathbf{Q}} / k$. It is, therefore, easy to see that parallel results also hold for a local number field $k_{v}$ and its algebraic closure $\bar{k}_{v}$, and for an algebraic number field $k$ and its maximal $p$-ramified $p$-extension $\tilde{k}^{(p)}$ when the Leopoldt conjecture holds for $k$ and $p$, because we have $H^{2}(\mathbb{S}, \mathbf{Q} / \mathbf{Z})$ $=0$ for $\mathbb{S}=\operatorname{Gal}\left(\bar{k}_{v} / k_{v}\right)$ and for $\mathbb{S}=\operatorname{Gal}\left(\tilde{k}^{(p)} / k\right)$, e.g. [He] or $[\mathrm{Ng}]$ for the latter case.

\section{REFERENCES}

[B-E] N. Blackburn and L. Evens, Schur multipliers of p-groups, J. reine angew Math., 309 (1979), 100-113.

[E-S] S. Eilenberg and N. Steenrod, Foundations of Algebraic Topology, Princeton U.P., Princeton, 1952.

[Fu] Y. Furuta, A norm residue map for central extensions of an algebraic number field, Nagoya Math. J., 93 (1984), 61-69.

[He] F.-P. Heider, Zahlentheoretische Knoten unendlicher Erweiterungen, Arch. Math., 37 (1981), 341-352.

[Iy] S. Iyanaga (ed.), The Theory of Numbers, North-Holland, Amsterdam-Oxford, and Amer. Elsevier, New York, 1975.

[Ka] G. Karpilovsky, The Schur Multiplier, Clarendon Press, Oxford, 1987.

[Kt] S. Katayama, On the Galois cohomology groups of $C_{k} / D_{k}$, Japan. J. Math., 8 (1982), 407-415.

[Ko] H. Koch, Galoissche Theorie der $p$-Erweiterungen, Springer-Verlag, Berlin-Heidelberg-New York, 1970. 
[Mi] K. Miyake, Central extensions and Schur's multiplicators of Galois groups, Nagoya Math. J., 90 (1983), 137-144.

[Ng] T. Nguyen-Quang-Do, Sur la structure galoisienne des corps locaux et la theorie d'Iwasawa, Compos. Math., 46 (1982), 85-119.

[Se] J. P. Serre, Modular forms of weight one and Galois representations, in: Algebraic number fields, ed. by A. Fröhlich, Ac. Press, London, 1977.

[Sh] S. S. Shatz, Profinite Groups, Arithmetic, and Geometry, Princeton U.P., Princeton, 1972 .

\section{Katsuya Miyake}

Department of Mathematics

College of General Education

Nagoya University

Nagoya, 464-01

Japan

Hans Opolka

Mathematisches Institut

Universität Göttingen

Bunsenstrasse 3-5

D-3400 Göttingen

B.R.D.

Present address:

Institut für Algebra und Zahlentheorie

Technische Universität Braunschweig

Pockelsstraße 14

$D$-W-3300 Braunschweig

B.R.D. 